\title{
Comparison of Canal Transportation in TruNatomy, ProTaper Gold, and Hyflex Electric Discharge Machining File Using Cone-beam Computed Tomography
}

\author{
Mukesh Kumar ${ }^{1}$, Ajay Paliwal ${ }^{2}$, Kumar Manish ${ }^{3}$, Sai K Ganapathy ${ }^{4}$, Neha Kumari ${ }^{5}$, Ayush R Singh ${ }^{6}$
}

\begin{abstract}
Aim: This study was carried out to compare canal transportation in three new rotary file systems, namely TruNatomy, ProTaper Gold, and Hyflex Electric Discharge Machining file system, using cone-beam computed tomography.

Materials and methods: The study was conducted on 105 extracted teeth consisting of 60 extracted mandibular first molars and 45 mandibular second premolars involving the mesiolingual canal of mandibular first molar and the root canal of second premolar. The teeth were randomly divided into three groups of 35 out of which each group consisted of 20 mandibular first molars and 15 mandibular second premolars. The TruNatomy rotary file was used in group I, ProTaper Gold was used in group II, and Hyflex Electric Discharge Machining was used in group III. Cone-beam computed tomography scan images were obtained both before and after instrumentation. Changes caused by preparation in the coronal, middle, and apical thirds were determined on cone-beam computed tomography scans and analyzed using the Kruskal-Wallis test at $\mathrm{p} \leq 0.05$ level of significance.

Results: TruNatomy showed least amount of canal transportation as compared with other two file systems at all the three levels of canals. ProTaper Gold showed maximum amount of canal transportation as compared with other two file systems at all the three levels of canals. The Hyflex EDM rotary file system showed transportation, which was more than the TruNatomy file system while it was less than that of ProTaper Gold. The difference was statically significant at the middle one-third level $(p=0.03)$ and at the coronal level $(p=0.02)$.

Conclusion: The TruNatomy system has least amount of canal transportation as compared with ProTaper Gold and Hyflex EDM system. With potential to preserve tooth structure, this file has an added advantage over other rotary file systems.

Clinical significance: The TruNatomy file system has been recently introduced with good cyclic fatigue resistance. It is necessary to evaluate the root canal transportation caused by the TruNatomy file.

Keywords: Canal transportation, Cone-beam computed tomography, Hyflex EDM, ProTaper Gold, TruNatomy.

The Journal of Contemporary Dental Practice (2021): 10.5005/jp-journals-10024-2970
\end{abstract}

\section{INTRODUCTION}

Biomechanical preparation of the root canal is the process carried out with the main aim of removing remnants of pulp, microorganisms, debris, and the necrotic tissue from the root canal and make it prepared for three-dimensional root canal filling. The canal preparation should be such that there is preservation of anatomy of the root canal alongwith the maintenance of the apical foramen position and prevention of weakening of the root canal. ${ }^{1}$ However, canal transportation is found during root canal preparation on application of rotary as well as manual instruments for root canal preparation. Canal transportation is the defect involving the dentin removal from the wall of the root canal. It is due to the property of the rotary or manual file to get back to its original shape and get in straight position during root canal preparation. ${ }^{2}$

It has been found that nickel titanium (NiTi) rotary files usually are found to preserve the anatomy of the root canal properly as compared with the contemporary stainless steel hand instruments. But root canal preparation of curved canals is still a major problem for the endodontists. ${ }^{3}$ Therefore, the manufacturers have introduced new designs and technologies of the rotary file system in order to improve their safety, performance, and mechanical properties. These features include controlled memory, thermal technology, $R$ phase and $M$ wire, and few more. Hyflex EDM (HFEDM), ProTaper Gold (PTG), and TruNatomy (TRN) file system are examples of the latest generation of rotary instruments. ${ }^{4,5}$
${ }^{1}$ Department of Conservative Dentistry and Endodontics, Patna Dental College and Hospital, Patna, Bihar, India

2,6 Department of Conservative Dentistry and Endodontics, Saraswati Dental College, Lucknow, Uttar Pradesh, India

${ }^{3}$ Department of Dentistry, Anugrah Narayan Medical College and Hospital, Gaya, Bihar, India

${ }^{4}$ Department of Conservative and Endodontics, Institute of Dental Studies and Technologies, Kadrabad, Modinagar, Ghaziabad, Uttar Pradesh, India

${ }^{5}$ Pediatric and Preventive Dentistry, Private Practitioner, Patna, Bihar, India Corresponding Author: Ajay Paliwal, Department of Conservative Dentistry and Endodontics, Saraswati Dental College, Lucknow, Uttar Pradesh, India, Phone: +91 8756852523, e-mail: drajaypaliwal@gmail.com How to cite this article: Kumar M, Paliwal A, Manish $K$, et al. Comparison of Canal Transportation in TruNatomy, ProTaper Gold, and Hyflex Electric Discharge Machining File Using cone-Beam Computed Tomography. J Contemp Dent Pract 2021;22(2):117-121.

Source of support: Nil

Conflict of interest: None

Hyflex EDM (Coltene, USA) is a new rotary file system that was launched with a new manufacturing property based on electric discharge machining popularly stated as the EDM-based file system. This file system has added advantage of controlled memory (CM)

(c) Jaypee Brothers Medical Publishers. 2021 Open Access This article is distributed under the terms of the Creative Commons Attribution 4.0 International License (https://creativecommons.org/licenses/by-nc/4.0/), which permits unrestricted use, distribution, and non-commercial reproduction in any medium, provided you give appropriate credit to the original author(s) and the source, provide a link to the Creative Commons license, and indicate if changes were made. The Creative Commons Public Domain Dedication waiver (http://creativecommons.org/publicdomain/zero/1.0/) applies to the data made available in this article, unless otherwise stated. 
treatment. Therefore, this rotary file system is believed to have improved flexibility and increased resistance to cyclic fatigue. Besides, there is reduction in the shape memory helping in reducing the canal defects. ${ }^{6,7}$ ProTaper Gold (PTG, Dentsply Sirona, Switzerland) is also a new rotary file system being manufactured from M-wire, which is new alloy of NiTi involving thermal treatment. It results in increasing the flexibility and cyclic fatigue resistance of PTG file system. ${ }^{8,9}$ TruNatomy instruments (TRN, Dentsply Sirona Switzerland) are the latest rotary file system being introduced with a special mechanical design and better heat treatment of $\mathrm{NiTi}$ alloys. It is available in three different shapes, which according to manufacturers creates a thin shaping leading to greater space in the canal. These are of parallelogram outline as observed in crosssection with off-centered design. As a result of this unique design, there is much improved flexibility of the TRN file system. ${ }^{10-12}$

No study has been conducted before to compare canal transportation in these three file systems. There can be various methods for this analysis but cone-beam computed tomography (CBCT) imaging is the most reliable technique because of its precise three-dimensional analysis of the root canal anatomy. Since the accuracy of CBCT is well established, therefore it is considered as a standard method for making comparison among different file systems for canal transportation. ${ }^{13}$

Hence, this study has been conducted to compare canal transportation in three new rotary file system, namely PTG, HFEDM), and TRN file system using CBCT.

\section{Materials and Methods}

The study was conducted at Patna Dental College, Patna. It was performed on 105 extracted teeth consisting 60 extracted mandibular first molars and 45 mandibular second premolars involving the mesiolingual canal of mandibular first molar and the root canal of mandibular second premolar. Only those teeth were included in the study in which there was one apical foramen with no signs of internal resorption of tooth. Those teeth that were found to have these defects were not included in the study and new normal teeth were used in place of them. There were orthodontic and periodontal reasons for the extraction of teeth. Mandibular first molar was selected for the study because it is the most common to undergo endodontic treatment. The collected teeth were treated with $0.1 \%$ thymol solution for 24 hours at $9^{\circ} \mathrm{C}$. The purpose of such process was to remove the tissue remnants and debris alongwith proper disinfection. Tap water was then used to remove the residues of thymol from the teeth. Then they were put in normal saline maintained at $4^{\circ} \mathrm{C}$. The teeth were stored until the analysis was carried out. Digital radiographs were taken for the initial analysis of root morphology using dental RVG (Vatech, Korea). All teeth were analyzed to rule out root defects like fracture, craze lines, and cracks with the help of a stereomicroscope adjusted at magnification of $\times 12$. The length of each tooth specimen was kept at a standard length of $19 \pm 1 \mathrm{~mm}$. It was done by using diamond discs for decapitation of tooth crown from the occlusal surface. The rotary file systems used in this study were TRN file (Densply Sirona, Switzerland), PTG file (Densply Sirona, Switzerland), and HFEDM file (Coltene, USA).

The teeth were randomly divided into three groups of 35 out of which each group consisted of 20 mandibular first molars and 15 mandibular second premolars. The TRN rotary file was used in group I. ProTaper Gold was used in group II, and HFEDM was used in group III. For TRN instruments, small size with 20/0.04 taper and prime size with 26/0.04 taper were used. ProTaper Gold was used in the following manner: SX file (one half of the working length), S1 file, S2 file, F1 and F2 files (full Working Length) with a taper of 25/0.08; HFEDM (25/0.08) was used for shaping the root canal up to working length according to the manufacturer's guidelines.

Before starting the root canal preparation, CBCT machine (Vatech 3D system, Korea) was used to obtain three-dimensional images. The exposure parameters were adjusted at exposure time of 10 seconds, current at $5.4 \mathrm{Ma}$, voxel size of $0.08 \mathrm{~mm}, \mathrm{kVp}$ at 89 , and field of view at $50 \times 50 \mathrm{~mm}$.

Preparation of the access cavity of each tooth was done with the help of a high-speed handpiece using diamond burs under water and air spray. Then there was negotiation of mesiolingual canal orifice of mandibular first molar and mandibular second premolar canal orifice. A \#10 K file (Densply) was used for determination of working length. It was introduced inside the root canal till its tip reached to the root apex. After that determination of working length took place, which was $1 \mathrm{~mm}$ less than the length measured with the $\# 10 \mathrm{~K}$ file. The instrumentation of all canals was done up to the determined working length by applying the crown down technique with the help of a endomotor. The endomotor used in this study was X-Smart plus Endomotor (Dentsply Maillefer, USA). The speed and torque were adjusted as per the instructions recommended by the manufacturer. $2.5 \%$ sodium hypochlorite solution was used for irrigation and $17 \%$ EDTA was used for removal of the smear layer.

The CBCT scans of the teeth were obtained after instrumentation with the same parameters used earlier for taking $C B C T$ scans before instrumentation (Fig 1). The wall thickness of the root canal was measured both before instrumentation as well as after instrumentation at three locations, which were at apical level, middle level, and coronal level by the same investigator who was kept completely unaware of the study design. All the root canal preparation was carried out by the same clinician.

\section{Canal Transportation}

Canal transportation was calculated in the mesiodistal direction (Fig. 2). Different measurements were taken in consideration. $\mathrm{X} 1$ represents the thickness of dentin before instrumentation while $\mathrm{X} 2$ represents the thickness after instrumentation. $\mathrm{Y} 1$ represents the distance between the periphery of the uninstrumented canal and furcation of the root. Y2 represents the distance between the periphery of the instrumented canal and the inside of the root. Measurements were done in both images of pre-instrumentation as well as post-instrumentation at three locations at 3, 6, and 9 $\mathrm{mm}$ from apex. Canal transportation at each level was calculated by using the method prescribed by Gambill et al. (Fig. 2$)^{14}$ with the help of equation: $[(\mathrm{X} 1-\mathrm{X} 2)-(\mathrm{Y} 1-\mathrm{Y} 2)] .{ }^{13}$ When the transportation value is zero, then it represents that there is no transportation; when the value is a positive then it represents that transportation is directed toward the mesial side; while the negative value represents that transportation is directed to the distal side of the canal. Data were collected and the statistical analysis was carried out by using the nonparametric Kruskal-Wallis test. $p \leq 0.05$ was considered as statistically significant.

\section{Results}

When evaluation was done at the apical one-third $(3 \mathrm{~mm}$ from root apex), then the TRN rotary file system showed minimum 


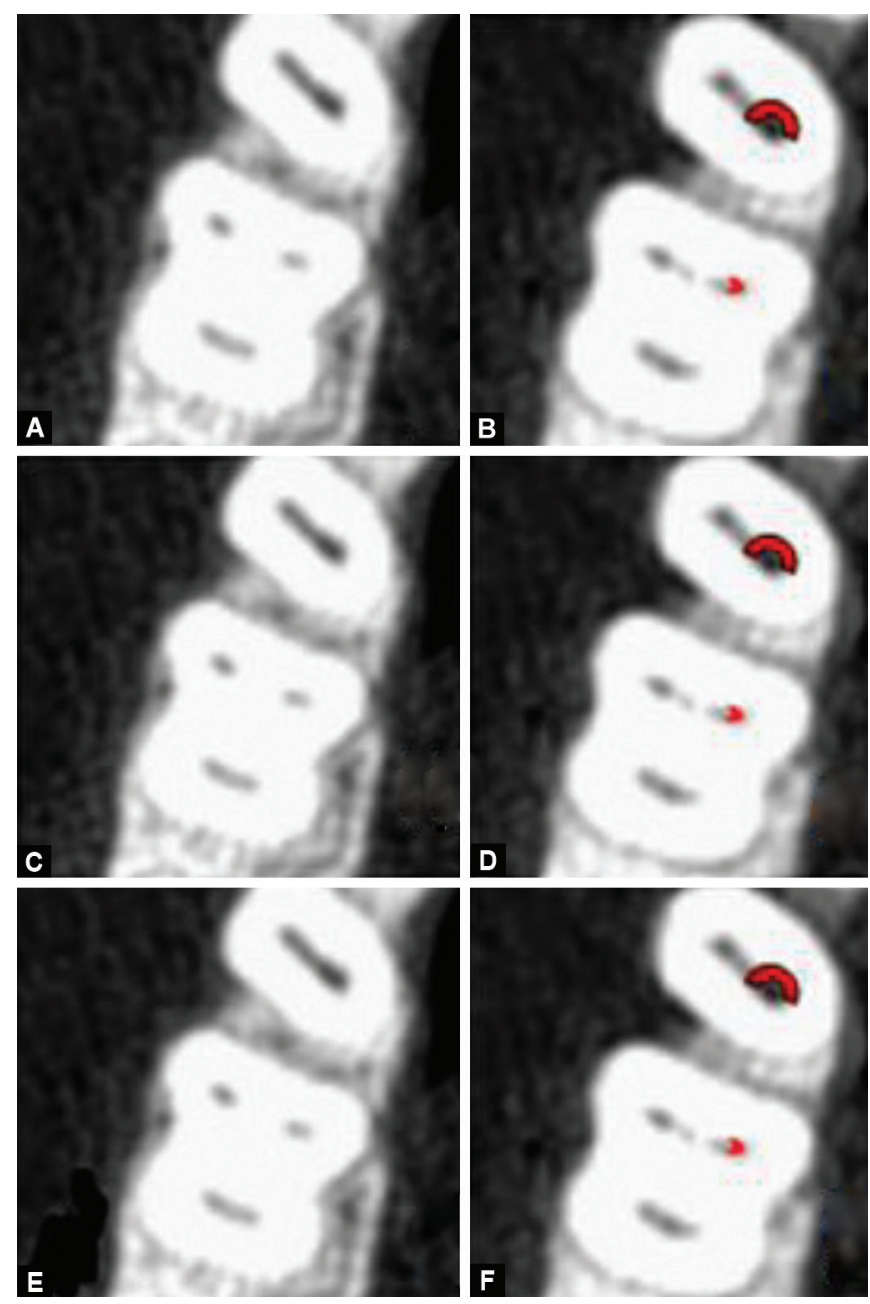

Figs $1 \mathrm{~A}$ to $\mathrm{F}$ : $(\mathrm{A})$ CBCT scan image of mandibular first molar and mandibular second premolar before instrumentation at apical level; (B) CBCT scan image after instrumentation at apical level; (C) Before instrumentation at middle one-third level; (D) After instrumentation at middle one-third level; (E) Before instrumentation at coronal one-third level; (F) After instrumentation at coronal one-third level. Red marks indicate canal transportation

transportation of $0.011 \pm 0.006 \mathrm{~mm}$ while PTG showed maximum mean transportation of $0.016 \pm 0.004 \mathrm{~mm}$. HFEDM showed transportation of $0.014 \pm 0.005 \mathrm{~mm}$ (Table 1 and Fig. 3).

During the analysis at the middle one-third $(6 \mathrm{~mm}$ from root apex), the TRN file system was found to have produced least transportation of $0.012 \pm 0.004 \mathrm{~mm}$ while PTG produced maximum transportation of $0.056 \pm 0.006 \mathrm{~mm}$. The HFEDM rotary file system showed transportation of $0.044 \pm 0.008 \mathrm{~mm}$, which was more than that of the TRN file system while it was less than that of PTG. The difference was statically significant with $p$ value $0.03(p \leq 0.05)$ (Table 1 and Fig. 3).

When mean amount of canal transportation by three file systems was analyzed at the coronal one-third ( $9 \mathrm{~mm}$ from apex), then the mean amount of transportation for the TRN file system was minimum, i.e., $0.023 \pm 0.002 \mathrm{~mm}$, while it was maximum in case of the PTG file system, i.e., $0.106 \pm 0.007 \mathrm{~mm}$. The mean amount of transportation for the HFEDM file system was $0.104 \pm 0.007 \mathrm{~mm}$. The difference was statically significant with $p$ value of $0.02(p \leq 0.05)$.

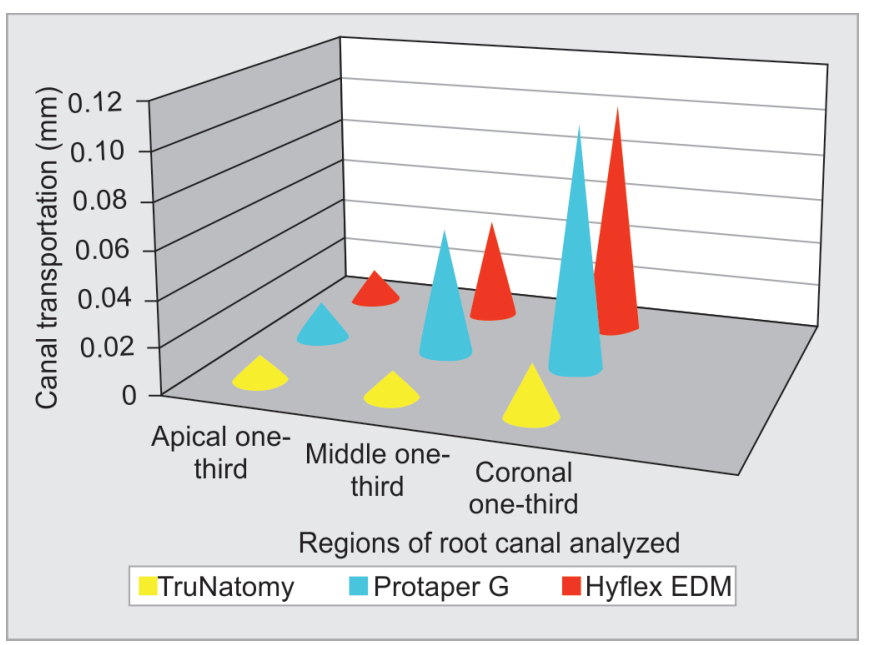

Fig. 2: Canal transportation by three file systems at three regions of canals

Table 1: Mean amount of canal transportation by three file systems at the apical, middle one-third, and coronal one-third levels

\begin{tabular}{|c|c|c|c|}
\hline Location & File system & $\begin{array}{l}\text { Mean amount of } \\
\text { transformation } \\
(\mathrm{mm})\end{array}$ & $p$ value \\
\hline \multirow[t]{3}{*}{ Apical one-third } & TRN & $0.011 \pm 0.006$ & 0.07 \\
\hline & PTG & $0.016 \pm 0.004$ & \\
\hline & HFEDM & $0.014 \pm 0.005$ & \\
\hline \multirow[t]{3}{*}{ Middle one-third } & TRN & $0.012 \pm 0.004$ & $0.03 *$ \\
\hline & PTG & $0.056 \pm 0.006$ & \\
\hline & HFEDM & $0.044 \pm 0.008$ & \\
\hline \multirow[t]{3}{*}{ Coronal one-third } & TRN & $0.023 \pm 0.002$ & $0.02 *$ \\
\hline & PTG & $0.106 \pm 0.007$ & \\
\hline & HFEDM & $0.104 \pm 0.005$ & \\
\hline
\end{tabular}

*Statistically significant

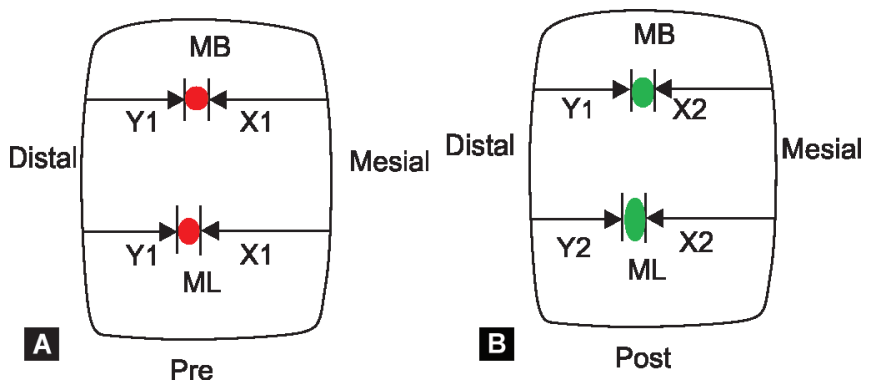

Figs $3 \mathrm{~A}$ and $\mathrm{B}$ : (A) Representative $\mathrm{CBCT}$ image of pre-instrumentation; (B) Representative $C B C T$ image of post-instrumentation. The measurement of canal transportation is done by using the equation prescribed by Gambill et al.: [(X1 - X2) - (Y1 - Y2)]

The TRN rotary file system showed minimum transportation while PTG showed maximum mean canal transportation. The HFEDM rotary file system showed transportation, which was more than the TRN file system while it was less than that of PTG. The results were statistically significant at the middle one-third and coronal level but at apical level the difference was not statistically significant. 


\section{Discussion}

It was observed in the current study that the TRN file system showed least transportation as compared with PTG and HFEDM rotary file system. The results were statistically significant at the middle one-third and coronal level but at the apical level the difference was not statistically significant. Since no study has been carried out to compare the TRN file system, HFEDM, and PTG, therefore this study was carried out with the purpose of evaluating canal transportation in these three new rotary file systems. Similar results were found when Elnaghy et al. conducted a study to compare the TRN file system with HyFlex CM (HCM), Vortex Blue (VB), and RaCe (RC) instruments. It was observed that the TRN file system was found to have better cyclic resistance to fracture. ${ }^{15}$ Riyahi et al. conducted a study to compare the cyclic resistance of the TRN file system with Twisted file and Protaper next rotary file system and concluded that the TRN file system showed better cyclic resistance as compared with other file systems. ${ }^{16}$ Vyver et al. presented a paper in which the authors described the advantages, clinical guidelines, and applications of the recently launched TRN system. It was concluded that TRN instruments cause proper preservation of dentine alongwith superior debridement. Preservation of the original anatomy of the root canal is also observed. ${ }^{17}$

In our study, canal transportation in case of HFEDM was less than PTG but more than that of TRN. Similar results were found when Turkistani et al. conducted a study to compare shaping ability of HFEDM and ProTaper Next rotary instruments in curved root canals by using micro-CT and found that HFEDM has better shaping ability than the Protaper Next rotary file system. ${ }^{18}$ Gagliardi et al. conducted a study to compare the canal transportation of PTG, ProTaper NEXT, and ProTaper Universal in curved canals and found that PTG and ProTaper Next caused less canal transportation as compared with ProTaper Universal. ${ }^{19}$ In the present study, the results were not similar as when PTG was compared with TRN and HEDM and it was found that canal transportation was more in case of PTG as compared with TRN and HEDM.

The main purpose of root canal instrumentation is preparation of the root canal in a shape such that it receive obturation as threedimensional root canal filling. However, there must be preservation of basic anatomy of the root canal. But many structural defects can take place during biomechanical preparation of root canals. One of such defects is canal transportation, which is actually a condition in which excessive dentin get removed from the walls of the root canal during root canal preparation. This condition arises due to the fact that files used in root canal preparation have a tendency to get back to their original shape and become straight during canal shaping. There may be formation of ledge, which may further lead to perforation of the canal causing failure of treatment. ${ }^{20}$

In the recent years, several $\mathrm{NiTi}$ instruments have been introduced, which are able to perform better root canal preparation maintaining the original anatomy of the canal in comparatively less time. ${ }^{21}$ Some of these new rotary file systems include TRN file system, PTG file system, HFEDM, etc. TruNatomy instruments (Dentsply Sirona) are the most recent rotary file system being introduced in the market with a special design and being made up of heat-treated NiTi alloy. ${ }^{15}$ These TRN are being available in three different sizes. This include small-size file system with taper of 20/0.4, prime-size file system with a taper of 26/0.04, and medium-size file system with a taper of $36 / 0.4$. According to the manufacturer, the three shaping instruments of TRN collectively provide a better debridement of dentine because of their unique slim design. This unique slim design of TRN provides sufficient space for the file system in the root canal for process of debridement. ${ }^{16}$ The unique slim design of the rotary file system is due to the reason that the NiTi wire used in TRN is of $0.8 \mathrm{~mm}$ design instead of $1.2 \mathrm{~mm}$ design being used commonly by other rotary NiTi file systems. The basic geometric design of the TRN file system is special parallelogram design along with an off-centered design. The TRN is being manufactured by utilizing special NiTi wire that is heattreated in an effective manner. This has caused enhancement in the flexibility of the TRN instrument. ${ }^{17}$

Due to all these properties, TRN is believed to maintain the basic anatomy of the root canal and preserve the integrity of tooth. The probability of developments of procedural defects like canal transportation is quite low in TRN. ${ }^{17}$ These file systems are available in the market in presterilized and prepacked forms, which are specially designed for shaping of the root canal with continuous taper design. This file system preserves the dentine of walls of the root canal. Therefore, the clinician is able to have TRN as a new simple and safe file system with increased cutting ability and better mechanical properties in comparison to previous rotary instruments meant for root canal preparation. ${ }^{15}$

In this study, a new three-dimensional imaging technique $\mathrm{CBCT}$ was used for analyzing the canal transportation. There can be other various methods for this analysis but $C B C T$ imaging is the most reliable technique because of its precise three-dimensional analysis of the root canal anatomy. Since the accuracy of CBCT is well-established, therefore it is considered as a standard method for making comparison among different file systems for canal transportation. . $^{141}$

The limitation of this study was that there was measurement of transportation in the mesial and distal directions only while the transportation in the buccal and lingual directions was deleted. There was heavy expenditure in this study, which led to limitation of the sample size.

\section{Conclusion}

The TRN system has least amount of canal transportation as compared with PTG and HFEDM systems. With this property to preserve tooth structure, this file has benefit over other rotary file systems. However, more studies are required to analyze other properties of this file system.

\section{References}

1. Peters OA. Current challenges and concepts in the preparation of root canal systems: a review. J Endod 2004;30(8):559-667. DOI: 10.1097/01.DON.0000129039.59003.9D.

2. Wei Z, Cui Z, Yan P, et al. A comparison of the shaping ability of three nickel-titanium rotary instruments: a micro-computed tomography study via a contrast radiopaque technique in vitro. BMC Oral Health 2017;17(1):39. DOI: 10.1186/s12903-016-0326-5.

3. Pereira ÉS, Viana AC, Buono VT, et al. Behavior of nickel-titanium instruments manufactured with different thermal treatments. J Endod 2015;41(1):67-71. DOI: 10.1016/j.joen.2014.06.005.

4. Alrahabi M. Comparative study of root-canal shaping with stainless steel and rotary NiTi files performed by preclinical dental students. Technol Health Care 2015;23(3):257-265. DOI: 10.3233/THC-150895.

5. Hülsmann M, Peters OA, Dummer PMH. Mechanical preparation of root canals: shaping goals, techniques and means. Endod Topics 2005;10(1):30-76. DOI: 10.1111/j.1601-1546.2005.00152.x.

6. Ye J, Gao Y. Metallurgical characterization of M-Wire nickeltitanium shape memory alloy used for endodontic rotary 
instruments during low-cycle fatigue. J Endod 2012;38(1):105-107. DOI: 10.1016/j.joen.2011.09.028.

7. Gao Y, Gutmann JL, Wilkinson K, et al. Evaluation of the impact of raw materials on the fatigue and mechanical properties of ProFile Vortex rotary instruments. J Endod 2012;38(3):398-401. DOI: 10.1016/j.joen.2011.11.004.

8. Silva EJNL, Vieira VCG, Tameirão MDN, et al. Quantitative transportation assessment in curved canals prepared with an offcentered rectangular design system. Braz Oral Res 2016;30(1):e43. DOI: 10.1590/1807-3107BOR-2016.vol30.0043.

9. Ruddle CJ, Machtou P, West JD. The shaping movement: fifth-generation technology. Dent Today 2013;32(4):94, 96-9.

10. Zhao D, Shen $Y$, Peng B, et al. Micro-computed tomography evaluation of the preparation of mesiobuccal root canals in maxillary first molars with Hyflex CM, Twisted files, and K3 instruments. J Endod 2013;39(3):385-388. DOI: 10.1016/j.joen.2012.11.030.

11. Pirani C, lacono F, Generali L, et al. HyFlex EDM: superficial features, metallurgical analysis and fatigue resistance of innovative electro discharge machined NiTi rotary instruments. Int Endod J 2016;49(5):483-493. DOI: 10.1111/iej.12470.

12. Testarelli L, Plotino G, Al-Sudani D, et al. Bending properties of a new nickel-titanium alloy with a lower percent by weight of nickel. J Endod 2011;37(9):1293-1295. DOI: 10.1016/j.joen.2011.05.023.

13. Kaval ME, Capar ID, Ertas H. Evaluation of the cyclic fatigue and torsional resistance of novel nickel-titanium rotary files with various alloy properties. J Endod 2016;42(12):1840-1843. DOI: 10.1016/j. joen.2016.07.015.

14. Gambill JM, Alder M, Rio CE. Comparison of nickel-titanium and stainless steel hand-file instrumentation using computed tomography. J Endod 1996;22(7):369-375. DOI: 10.1016/S00992399(96)80221-4.

15. Elnaghy $\mathrm{AM}$, Elsaka $\mathrm{SE}$, Mandorah $\mathrm{AO}$. In vitro comparison of cyclic fatigue resistance of TruNatomy in single and double curvature canals compared with different nickel-titanium rotary instruments. BMC Oral Health 2020;20(1):38. DOI: 10.1186/ s12903-020-1027-7.

16. Riyahi AM, Bashiri A, Alshahrani K, et al. Cyclic fatigue comparison of TruNatomy, twisted file, and ProTaper next rotary systems. Int J Dent 2020;2020:3190938. DOI: 10.1155/2020/3190938.

17. Vyver PJ, Vorster $M$, Peters $O A$. Minimally invasive endodontics using a new single-file rotary system. Int Dent Afr 2019;9(4):6-20.

18. Turkistani AK, Gomaa MM, Shafei LA, et al. Shaping ability of HyFlex EDM and ProTaper next rotary instruments in curved root canals: a micro-CT study. J Contemp Dent Pract 2019;20(6):680-685. DOI: 10.5005/jp-journals-10024-2579.

19. Gagliardi J, Versiani MA, Neto MD, et al. Evaluation of the shaping characteristics of ProTaper gold, ProTaper NEXT, and ProTaper universal in curved canals. J Endod 2015;41(10):1718-1724. DOI: 10.1016/j.joen.2015.07.009.

20. Al-Sudani D, Grande NM, Plotino G, et al. Cyclic fatigue of nickel-titanium rotary instruments in a double (S-shaped) simulated curvature. J Endod 2012;38(7):987-989. DOI: 10.1016/j. joen.2012.03.025.

21. Paqué $F$, Barbakow F, Peters OA. Root canal preparation with Endo-Eze AET: changes in root canal shape assessed by microcomputed tomography. Int Endod J 2005;38(7):456-464. DOI: 10.1111/j.1365-2591.2005.00968.x. 